University of Nebraska - Lincoln

DigitalCommons@University of Nebraska - Lincoln

$4-1-2006$

\title{
Relative stability of planar versus double-ring tubular isomers of neutral and anionic boron cluster $\mathrm{B}_{20}$ and $\mathrm{B}_{20}{ }^{-}$
}

Wei An

University of Nebraska-Lincoln

Satya S. Bulusu

University of Nebraska-Lincoln, sbulusu@iiti.ac.in

Yi Gao

University of Nebraska-Lincoln, ygao3@Unl.edu

Xiao Cheng Zeng

University of Nebraska-Lincoln, xzeng1@unl.edu

Follow this and additional works at: https://digitalcommons.unl.edu/chemzeng

Part of the Chemistry Commons

An, Wei; Bulusu, Satya S.; Gao, Yi; and Zeng, Xiao Cheng, "Relative stability of planar versus double-ring tubular isomers of neutral and anionic boron cluster $\mathrm{B}_{20}$ and $\mathrm{B}_{20}{ }^{-1}$ (2006). Xiao Cheng Zeng Publications. 10.

https://digitalcommons.unl.edu/chemzeng/10

This Article is brought to you for free and open access by the Published Research - Department of Chemistry at DigitalCommons@University of Nebraska - Lincoln. It has been accepted for inclusion in Xiao Cheng Zeng Publications by an authorized administrator of DigitalCommons@University of Nebraska - Lincoln. 


\title{
Relative stability of planar versus double-ring tubular isomers of neutral and anionic boron cluster $\mathrm{B}_{20}$ and $\mathrm{B}_{20}^{-}$
}

\author{
Wei An, Satya Bulusu, Yi Gao, and X. C. Zeng ${ }^{\text {a) }}$ \\ Department of Chemistry, University of Nebraska-Lincoln, Lincoln, Nebraska 68588
}

(Received 13 January 2006; accepted 21 February 2006; published online 19 April 2006)

\begin{abstract}
High-level $a b$ initio molecular-orbital methods have been employed to determine the relative stability among four neutral and anionic $\mathrm{B}_{20}$ isomers, particularly the double-ring tubular isomer versus three low-lying planar isomers. Calculations with the fourth-order Møller-Plessset perturbation theory [MP4(SDQ)] and Dunning's correlation consistent polarized valence triple zeta basis set as well as with the coupled-cluster method including single, double, and noniteratively perturbative triple excitations and the $6-311 \mathrm{G}(d)$ basis set show that the double-ring tubular isomer is appreciably lower in energy than the three planar isomers and is thus likely the global minimum of neutral $\mathrm{B}_{20}$ cluster. In contrast, calculations with the MP4(SDQ) level of theory and 6-311 $+\mathrm{G}(d)$ basis set show that the double-ring anion isomer is appreciably higher in energy than two of the three planar isomers. In addition, the temperature effects on the relative stability of both ${ }^{10} \mathrm{~B}_{20}^{-}$ and ${ }^{11} \mathrm{~B}_{20}^{-}$anion isomers are examined using the density-functional theory. It is found that the three planar anion isomers become increasingly more stable than the double-ring isomer with increasing the temperature. These results are consistent with the previous conclusion based on a joint experimental/simulated anion photoelectron spectroscopy study [B. Kiran et al., Proc. Natl. Acad. Sci. U.S.A. 102, 961 (2005)], that is, the double-ring anion isomer is notably absent from the experimental spectra. The high stability of the double-ring neutral isomer of $\mathrm{B}_{20}$ can be attributed in part to the strong aromaticity as charaterized by its large negative nucleus-independent chemical shift. The high-level ab initio calculations suggest that the planar-to-tubular structural transition starts at $B_{20}$ for neutral clusters but should occur beyond the size of $B_{20}^{-}$for the anion clusters.

(C) 2006 American Institute of Physics. [DOI: 10.1063/1.2187003]
\end{abstract}

\section{INTRODUCTION}

Boron, the first light element of group IIIA with one $p$ valence electron, is known as a semiconductor element. Because of its short covalent radius, electron deficiency, and $s p^{2}$ hybridization of the valence electron, boron tends to form strong and directional covalent bond with other elements. As a consequence, boron can form diverse nanostructures. So far, four topological forms of nanostructures, including quasiplanar, tubular, convex, and spherical, have been identified. ${ }^{1-3}$ As an intermediate regime between an atom and bulk phase, it is well known that nanoscale materials can exhibit novel structures whose physical, chemical, and electronic properties can be remarkably different from those of their bulk counterparts. Examples include atomic clusters and nanotubes of carbon, silicon, and gold. ${ }^{4-6}$

Bulk boron has demonstrated some of interesting chemical versatility compared to its neighboring elements, such as a very high melting point of $2349 \mathrm{~K}$ (nearly $700 \mathrm{~K}$ higher than that of silicon), a hardness close to diamond but very low density, and electronically semiconducting. These remarkable properties lead boron to potentially important applications such as high-temperature semiconductors, ${ }^{7}$ superconductor, $^{8}$ and high-energy density fuels. ${ }^{9,10}$ Over the past few years, boron nanostructures have been exten-

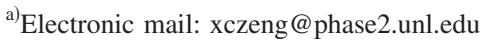

sively investigated both theoretically ${ }^{1-3,11-21}$ and experimentally. ${ }^{14-17}$ The growing research interests are largely driven by the technological challenges of miniaturization of electronic devices. Functioning as building blocks for self-assembled material, boron nanostructures can have potential applications in high-performance nanoscale devices.

Bulk boron exists in amorphous phase, as well as in $\alpha$ and $\beta$-rhombohedral and $\alpha$-tetragonal forms with unit cells all containing three-dimensional (3D) $\mathrm{B}_{12}$ icosahedra. ${ }^{22,23} \mathrm{In}$ contrast, two-dimensional (2D) planar and quasiplanar structures are the most stable structures of $\mathrm{B}_{n}$ clusters in the size range of $3 \leqslant n \leqslant 18 .^{2,11-17}$ Recently, a combined experimental/simulated photoelectron spectroscopy study suggests that a planar-to-tubular structural transition is likely to occur at $n=20$ (Ref. 17) and beyond $n=20,3 \mathrm{D} \mathrm{B}_{n}$ clusters become the energetically more stable structure. ${ }^{18,19}$ A better understanding of the structural evolution of atomic clusters will provide more insight into the growth pathway from individual atoms to solid-state material of an element. However, due to high reactivity of small-sized atomic clusters (probably existing only in gas-phase molecule beam) and the difficulties associated with numerical experimental techniques, addressing this issue has always been a challenging task.

In the previous study, ${ }^{17}$ we performed an unbiased global-minimum search combined with density-functional 
theory (DFT) calculations to seek the most stable structure of neutral $\mathrm{B}_{20}$ cluster. We showed that the double-ring tubular $\mathrm{B}_{20}$ isomer is the global minimum on basis of the DFT calculations, and that the double-ring tubular $\mathrm{B}_{20}^{-}$anion isomer is isoenergetic with two quasiplanar structures. We concluded that the threshold of 2D-to-3D transition occurs at $n$ $=20$ for the neutral boron clusters. On the other hand, the simulated anion photoelectron spectroscopy (PES) of the double-ring $\mathrm{B}_{20}^{-}$was found not matching the measured PES, suggesting that the double-ring $\mathrm{B}_{20}^{-}$isomer is unlikely to be the most stable anion structure, at least under the experimental conditions (above the room temperature). ${ }^{17}$ This apparent inconsistency between the theoretical prediction on the structure of neutral cluster (at $0 \mathrm{~K}$ ) and the experimental measurement of the anion cluster (at elevated temperatures) calls for more studies.

In this work, we will (1) reexamine the relative stability of the four low-lying, both neutral and anionic, $\mathrm{B}_{20}$ isomers (as studied in Ref. 17; see Fig. 1) by using high-level $a b$ initio molecular-orbital methods with reasonably large basis sets; (2) evaluate temperature effects on the relative stability of the four low-lying anionic isomers; and (3) investigate the aromaticity of the double-ring structure of the neutral $\mathrm{B}_{20}$.

\section{THEORETICAL METHODS}

All calculations were performed using Gaussian 03, Revision C.02 package. ${ }^{24}$ For neutral clusters, full geometry optimizations were performed using the second-order MøllerPlesset perturbation theory ${ }^{25,26}$ (MP2) method as well as DFT methods in generalized gradient approximations (GGAs) with two hybrid exchange-correlation functionals, namely, B3LYP (Ref. 27) and PBE1PBE, ${ }^{28}$ and a recently developed hybrid metafunctional TPSS1KCIS. ${ }^{29}$ A modest cc-pVDZ basis set ${ }^{30}$ [Dunning's correlation consistent polarized valence double zeta, contracted $[3 s 2 p]$ plus polarization set $(1 d)$ ] was chosen with the MP2 method and a large ccpVTZ basis set ${ }^{30}$ [Dunning's correlation consistent polarized valence triple zeta, contracted $[4 s 3 p]$ plus polarization set $(2 d 1 f)]$ with DFTs. Next, the harmonic vibrationalfrequency analyses were carried out to assure that the optimized structures give no imaginary frequencies. To determine the energy ordering, several high-level $a b$ initio molecular-orbital methods were employed to calculate single-point energies of the four neutral isomers with the optimized structures at the MP2/cc-pVDZ level of theory: (1) the fourth-order Møller-Plesset perturbation theory ${ }^{31}$ (MP4) with cc-pVTZ basis set for neutral isomers; (2) a coupled-cluster $^{32}$ method at the CCSD(T1Diag)/6-311G $(d)$ level of theory (to examine possible multireference quality for the top-two lowest-energy isomers); and (3) the coupledcluster method including single, double, and noniteratively perturbative triple excitations at the $\operatorname{CCSD}(\mathrm{T}) / 6-311 \mathrm{G}(d)$ level of theory.

For anion clusters, full geometry optimizations were performed using the DFT methods with the B3LYP and PBE1PBE functionals and the Pople-type 6-311+G $(d)$ basis set. Gibbs free energies of the four anionic $\mathrm{B}_{20}^{-}$isomers as a function of temperature (from $0.5 \mathrm{~K}$ to $2000 \mathrm{~K}$ ) were calcu-
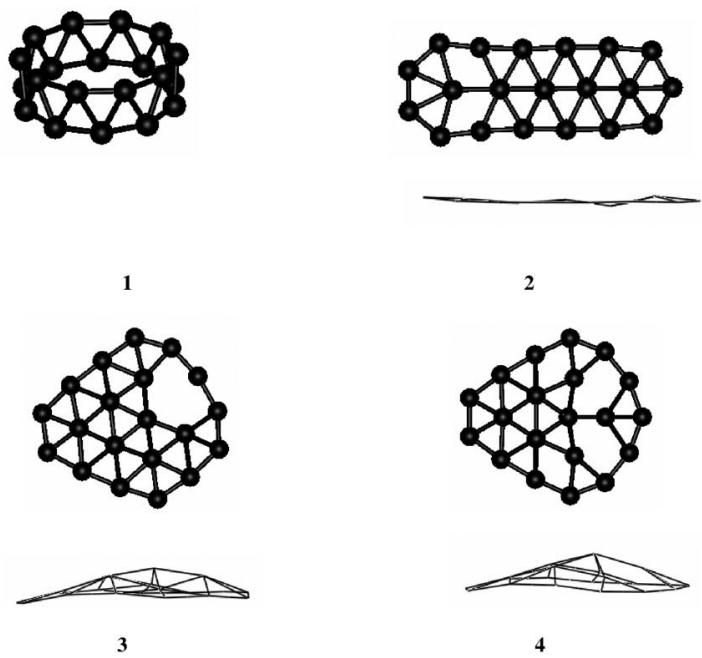

FIG. 1. The double-ring tubular (1) and three planar low-lying isomers (2), (3), and (4) of the neutral $\mathrm{B}_{20}$ cluster.

lated at the PBE1PBE/6-311+G $(d)$ level of theory. Lastly, an analysis of molecular-orbital nucleus-independent chemical shifts $^{33}$ (MO-NICSs) with the canonical molecular-orbital $(\mathrm{CMO})$ method (built in the NBO 5.0 program) ${ }^{34}$ was undertaken [at the PBE1PBE/6-311+G(d) and B3LYP/ $6-311+\mathrm{G}(d)$ levels of theory, respectively] to investigate aromaticity of the neutral tubular $\mathrm{B}_{20}$ cluster.

\section{RESULTS AND DISCUSSIONS}

\section{A. Relative stability among the four neutral isomers at $0 \mathrm{~K}$}

Relative stability (or energy ordering) among low-lying isomers is an important index to characterize a molecular or cluster system. Searching for the low-lying minima on the potential-energy surface for atomic structures is of fundamental importance to cluster physics and chemistry. There has been particular interest in locating the global minimum at $0 \mathrm{~K}$, since the growth pathway of atomic structures is likely related to the global-minimum structures. As a result of a global search combined with DFT calculations, the doublering tubular structure of $\mathrm{B}_{20}$ (Fig. 1) was identified as the leading candidate for the global minimum. ${ }^{17}$ Later, Marques and Botti ${ }^{20}$ also performed DFT calculations using the PBE exchange-correlation functional and confirmed that the double-ring tubular structure of $\mathrm{B}_{20}$ is the lowest-energy isomer, although the energy ordering among other low-lying isomers differs from ours derived from the B3LYP functional. The disparities in the energy ordering obtained from the two different functionals have been reported for many cluster systems, for example, carbon clusters and silicon clusters. ${ }^{35}$ A more accurate energy ordering can be obtained via high-level ab initio methods, such as the MP4, coupledcluster (CC), ${ }^{32,36}$ or configuration interaction (CI) method. ${ }^{37}$ However, the computational costs for these ab initio methods increase rapidly with the numbers of basis functions, which is directly related to the size of a molecular system and the number of electrons involved.

First, we employed the MP4 method together with a large basis set (cc-pVTZ) to calculate the single-point energy 
TABLE I. Calculated relative energy (in eV) of the three planar neutral $\mathrm{B}_{20}$ isomers (2), (3), and (4) with respect to the double-ring isomer (1) at various levels of theory.

\begin{tabular}{lcccc}
\hline \hline \multicolumn{1}{c}{ Method } & $1\left(D_{10 d}\right)$ & $2\left(C_{s}\right)$ & $3\left(C_{1}\right)$ & $4\left(C_{s}\right)$ \\
\hline MP4(SDQ)/cc-pVTZ//MP2/cc-pVDZ & 0.00 & 0.52 & 0.20 & 0.32 \\
CCSD(T1Diag)/6-311G(d)//MP2/cc-pVDZ & 0.00 & $\ldots$ & 0.69 & $\ldots$ \\
CCSD(T)/6-311G(d)//MP2/cc-pVDZ & 0.00 & 0.70 & 0.46 & 0.53 \\
PBE1PBE/cc-pVTZ & 0.00 & 0.96 & 0.66 & 0.80 \\
TPSS1KCIS/cc-pVTZ & 0.00 & 0.84 & 0.66 & 0.82 \\
B3LYP/cc-pVTZ & 0.00 & 1.00 & 0.84 & 1.01 \\
\hline \hline
\end{tabular}

for the four neutral $\mathrm{B}_{20}$ isomers (Fig. 1) optimized at the MP2/cc-pVDZ level. As shown in Table I, the energy ordering derived from the MP4(SDQ)/cc-pVTZ//MP2/cc-pVDZ calculation confirms that the double-ring structure 1 of $\mathrm{B}_{20}$ has the lowest energy among the four isomers considered, followed by $3(0.20 \mathrm{eV}$ higher in energy), $4(0.32 \mathrm{eV})$, and 2 $(0.52 \mathrm{eV})$. Next, single-point energy calculations at the CCSD(T1Diag)/6-311G(d)//MP2/cc-pVDZ level of theory were performed to examine possible multireference character of the two lowest-energy structures, i.e., 1 and 3. The isomer 1 yields a T1 diagnostic value of 0.014 , while 3 yields a larger value of 0.022 . A T1 value of 0.02 or greater indicates certain degree of multireference character for the molecular system and thus the energy calculation based on singlereference electron correlation methods may not be fully reliable. ${ }^{38}$ In this case, the more accurate multireference CI (MRCI) method would be a better choice to determine the relative stability between 1 and 3 . However, it is not feasible with our current computer capability to perform such calculations for a medium-sized molecular system like $\mathrm{B}_{20}$. Instead, we employed $\operatorname{CCSD}(\mathrm{T})$ (Ref. 36) method to further examine the energy ordering as the $\operatorname{CCSD}(\mathrm{T})$ calculation is an efficient MRCI approximation, and yet it is affordable to handle the $\mathrm{B}_{20}$ cluster. As shown in Table I, the $\operatorname{CCSD}(\mathrm{T}) /$ $6-311 \mathrm{G}(d)$ calculation again confirms that the tubular 1 is energetically the most stable structure followed by 3,4 , and 2 with $0.46,0.53$, and $0.70 \mathrm{eV}$ higher in energy than 1 , respectively. In Table $\mathrm{I}$, the energy orderings given by PBE1PBE/cc-pVTZ, B3LYP/cc-pVTZ, and TPSS1KCIS/ccpVTZ DFT calculations are also listed for comparison. It is interesting to note that the energy ordering based on the PBE1PBE functional agrees well with that based on the MP4 and $\operatorname{CCSD}(\mathrm{T})$ calculations. Similar conclusion was also reported for the carbon cluster $\mathrm{C}_{20} \cdot{ }^{35}$

On the experimental side, however, neutral $\mathrm{B}_{20}$ has yet to be synthesized. The anion PES is a reliable experimental protocol to characterize highly reactive atomic clusters ${ }^{17}$ which are usually observed in anionic or cationic state in the gas phase. For the four anionic $\mathrm{B}_{20}^{-}$isomers, we calculated their single-point energy using MP4(SDQ)/6-311 $+\mathrm{G}(d) / / \mathrm{PBE} 1 \mathrm{PBE} / 6-311+\mathrm{G}(d)$ level of theory. Interestingly, the energy ordering among the anions is opposite to the ordering among their neutral counterparts (see Table II), with 4 $(-0.59 \mathrm{eV})<3(-0.51 \mathrm{eV})<2(-0.01 \mathrm{eV})<1(0.00 \mathrm{eV})$. In particular, the two planar isomers 4 and 3 are appreciably lower in energy than the double-ring isomer 1; the planar 2 is isoenergetic with 1 . This energy ordering for the anion isomers supports the conclusion made from previous experimental/simulated PES study in that because the double-ring anion isomer 1 is not observed in the measured PES spectra, the most stable anion structures are still the planar isomers. ${ }^{17}$ The energy orderings obtained from PBE1PBE/6-311+ $\mathrm{G}(d)$ and B3LYP/6-311+ $\mathrm{G}(d)$ calculations are also listed in Table II for comparison. Again, the PBE1PBE functional yields more consistent energy ordering than the B3LYP functional, in comparison with the MP4 results.

\section{B. Temperature effects on free-energy ordering}

In reality, the PES measurements were performed with the ${ }^{10} \mathrm{~B}_{20}^{-}(99.75 \%)$ isotopic anions at temperatures above $300 \mathrm{~K}$ (and maybe up to $700 \mathrm{~K}$ ). ${ }^{17}$ To assess the temperature effects on the relative stability among the four low-lying anionic isomers, we performed a thermochemical analysis for the ${ }^{10} \mathrm{~B}_{20}^{-}$anionic isomers, as well as for ${ }^{11} \mathrm{~B}_{20}^{-}$(the most abundant form of boron in nature). The equations required for the themochemical analysis were described elsewhere. ${ }^{35}$

In Fig. 2, we plot relative free energies as a function of temperature (from 0.5 to $2000 \mathrm{~K}$ ), which were calculated based on the PBE1PBE/6-311+G $(d)$ level of theory for the four low-lying anionic isomers. The calculations show that the planar isomers 2,3 , and 4 become increasingly stable than the double-ring isomer 1 as temperature increases. It appears that the entropy contribution to the free energy favors the $2 \mathrm{D}$ planar structure over the $3 \mathrm{D}$ double-ring struc-

TABLE II. Calculated relative energy (in $\mathrm{eV}$ ) of the three planar anionic $\mathrm{B}_{20}^{-}$isomers with respect to the double-ring isomer at the three levels of theory. The boldfaced energy values highlight those planar isomers with lower energy than the double-ring isomer.

\begin{tabular}{lcrrr}
\hline \multicolumn{1}{c}{ Method } & $1\left(C_{2 v}\right)$ & $2\left(C_{s}\right)$ & $3\left(C_{1}\right)$ & $4\left(C_{s}\right)$ \\
\hline MP4(SDQ)/6-311+G(d)//PBE1PBE/6-311+G(d) & 0.00 & $-\mathbf{0 . 0 1}$ & $\mathbf{- 0 . 5 1}$ & $\mathbf{- 0 . 5 9}$ \\
PBE1PBE/6-311+G(d) & 0.00 & 0.11 & $\mathbf{- 0 . 0 5}$ & 0.02 \\
B3LYP/6-311+G(d) & 0.00 & 0.13 & 0.16 & 0.28 \\
\hline \hline
\end{tabular}




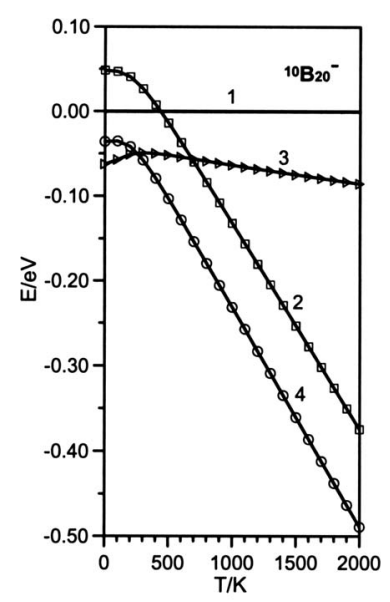

(a)

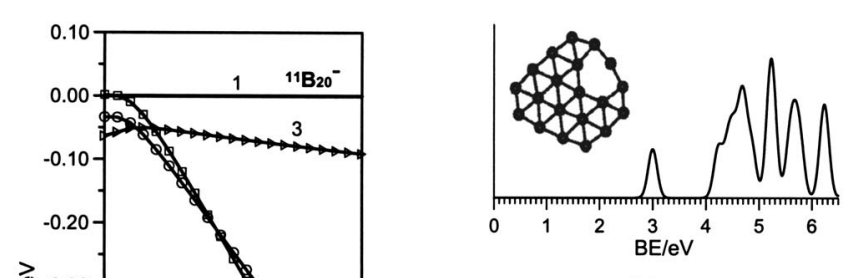

(a)

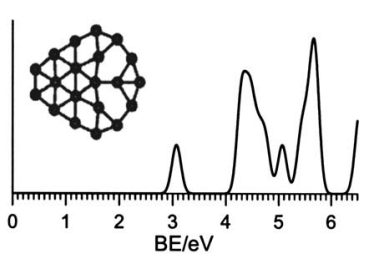

(b)
FIG. 3. Simulated anion photoelectron spectra for (a) 3 and (b) 4. The spectra were constructed by fitting each of the vertical detachment transitions with a Gaussian of $0.05 \mathrm{eV}$ width. BE denotes the binding energy (in $\mathrm{eV}$ ).

$n<16$, the 2D planar and quasiplanar geometries of boron clusters $\mathrm{B}_{n}$ are overwhelmingly stable. ${ }^{2,11-17}$ Such strong stability is attributed in part to the intrinsic strong aromaticity of the planar boron clusters. ${ }^{12,15,16,24}$ If the $\mathrm{B}_{20}$ cluster assumes the double-ring tubular geometry as the globalminimum, strong cylindrical aromaticity may be expected in the tubular geometry.

The NICSs can be used as an aromaticity indicator. ${ }^{33} \mathrm{~A}$ large negative or positive NICS value at positions with little electron density indicates strong aromaticity or antiaromaticity. In general, the Hückel $4 N+2$ rule can be applied to simple cyclic aromaticity, whereas the Hirsch's $2(N+1)^{2}$ rule can be related to the spherical/cylindrical aromaticity. ${ }^{39}$ However, the diversity of molecular geometries has made aromaticity more complex than previously expected. Very recently, one study showed that the Hückel $4 N+2$ rule of aromaticity may not be applicable to polycyclic $\pi$ systems such as the planar boron clusters. ${ }^{24}$

A full analysis of MO-NICS is important to the understanding of aromaticity of a polycyclic molecular system. ${ }^{40,41}$ We calculated MO-NICS at the center of the double-ring $\mathrm{B}_{20}$ isomer. In Table III, contributions from individual $\mathrm{MO}$ to the magnetic shielding of a ghost atom at the center of the double-ring $\mathrm{B}_{20}$ isomer are presented. Both PBE1PBE/6 $-311 \mathrm{G}(d)$ and B3LYP/6-311G $(d)$ levels of theory were used to compute the MO-NICS values. It can be seen that there is little difference between NICS values obtained from the two functionals. The large negative NICS value of -40 for the double-ring $\mathrm{B}_{20}$ compared to the smaller negative NICS value for benzene $(-8.0), \mathrm{B}_{8}^{2-}(-27.0)$, and $\mathrm{B}_{9}^{-}(-23.2)^{17}$ demonstrate the presence of strong $\sigma$ and $\pi$ aromaticities within the $\mathrm{B}_{20}$ structure. Note that the NICS value at the position close to the nucleus would be much higher (negative) than the value at the position away from the nucleus. The distance between the ghost atom and 20 boron atoms in the double-ring structure is about $2.7 \AA$ (the diameter of double-ring tubular is $5.2 \AA$ ). The NICS values for the planar clusters $\mathrm{B}_{8}^{2-}$ and $\mathrm{B}_{9}^{-}$were calculated $0.5 \AA$ above the center of the molecular plane. Hence, considering the position dependence of NICS value, the aromaticity of the neutral tubular $\mathrm{B}_{20}$ cluster is even more pronounced. The neutral $\mathrm{B}_{20}$ cluster 1 with $D_{10 d}$ symmetry has a closed electronic shell with highly degenerate electronic configuration (see Table II). The MOs from No. 21 to 32 and those close to the highest occupied molecular orbital (HOMO) from No. 42 to 48 constitute most of the diatropic contributions to aromaticity, while MOs of Nos. 33, 34, 38 to 41, and HOMOs contribute 
TABLE III. Calculated CMO-NICS (in ppm) for the neutral double-ring $\mathrm{B}_{20}$ cluster. The boldfaced values are discussed in the text.

\begin{tabular}{|c|c|c|c|}
\hline MO & MO label & CMO-NICS $^{\mathrm{a}, \mathrm{b}}$ & $\mathrm{CMO}^{-N I C S^{\mathrm{a}, \mathrm{c}}}$ \\
\hline 21 & $a_{1}$ & -3.78 & -3.92 \\
\hline 22 & $e_{1}$ & -4.21 & -4.18 \\
\hline 23 & $e_{1}$ & -4.21 & -4.18 \\
\hline 24 & $e_{2}$ & -3.95 & -3.87 \\
\hline 25 & $e_{2}$ & -3.95 & -3.87 \\
\hline 26 & $e_{2}$ & -3.00 & -2.97 \\
\hline 27 & $e_{2}$ & -3.00 & -2.97 \\
\hline 28 & $e_{1}$ & -1.81 & -1.76 \\
\hline 29 & $e_{1}$ & -1.82 & -1.76 \\
\hline 30 & $a_{1}$ & -3.85 & -3.60 \\
\hline 31 & $e_{1}$ & -2.79 & -2.60 \\
\hline 32 & $e_{1}$ & -2.79 & -2.60 \\
\hline 33 & $a_{2}$ & 2.51 & 2.52 \\
\hline 34 & $a_{1}$ & 2.51 & 2.52 \\
\hline 35 & $e_{2}$ & -1.56 & -1.50 \\
\hline 36 & $e_{2}$ & -1.56 & -1.50 \\
\hline 37 & $a_{1}$ & -4.72 & -4.79 \\
\hline 38 & $e_{2}$ & 2.47 & 2.41 \\
\hline 39 & $e_{2}$ & 2.47 & 2.41 \\
\hline 40 & $e_{1}$ & 9.83 & 9.67 \\
\hline 41 & $e_{1}$ & 9.83 & 9.67 \\
\hline 42 & $e_{1}$ & -1.85 & -1.89 \\
\hline 43 & $e_{1}$ & -1.85 & -1.89 \\
\hline 44 & $a_{1}$ & -4.72 & -4.51 \\
\hline 45 & $e_{1}$ & -2.74 & -2.64 \\
\hline 46 & $e_{1}$ & -2.74 & -2.64 \\
\hline 47 & $e_{2}$ & -0.04 & -0.18 \\
\hline 48 & $e_{2}$ & -0.03 & -0.17 \\
\hline 49 & $e_{2}$ & 0.35 & 0.61 \\
\hline 50 & $e_{2}$ & 0.34 & 0.60 \\
\hline Total & & -40.98 & -39.86 \\
\hline
\end{tabular}

${ }^{a}$ Only MO-NICS $\geqslant 1.00 \mathrm{ppm}$ listed except for HOMOs.

${ }^{\mathrm{b}} \mathrm{PBE} 1 \mathrm{PBE} / 6-311+\mathrm{G}(d)$.

${ }^{c} \mathrm{~B} 3 \mathrm{LYP} / 6-311+\mathrm{G}(d)$.

paratropically to aromoticity (highlighted in boldface in Table II). NICS is primarily determined by the motion of $\pi$ electrons; the $N$ in both formula $4 N+2$ and $2(N+1)^{2}$ represent the number of $\pi$ electrons. However, contributions from local circulations of electrons in $\sigma$ bonds, lone pairs, and even atom cores cannot be neglected. ${ }^{42,43}$ In other words, the entire molecular framework should be taken into account. The $s p^{2}$ hybridization, electron deficiency of boron in conjunction with the unique double-ring nuclear arrangement is responsible for the strong aromaticity and high stability of the neutral double-ring $\mathrm{B}_{20}$ cluster.

\section{CONCLUSIONS}

We have studied relative stability among four low-lying isomers of neutral and anionic $\mathrm{B}_{20}$ clusters. The MP4(SDQ) and $\operatorname{CCSD}(\mathrm{T})$ calculations yield the same energy ordering for the neutral $\mathrm{B}_{20}$ isomers, and both calculations show that the double-ring tubular structure 1 is the lowest-energy structure. In contrast, the MP4(SDQ) calculations show that the planar 4 is the lowest-energy structure while 1 is the highestenergy structure among the four anionic $\mathrm{B}_{20}^{-}$isomers. The trend of relative stability with increasing temperature for both ${ }^{10} \mathrm{~B}_{20}^{-}$and ${ }^{11} \mathrm{~B}_{20}^{-}$isomers suggests that the planar isomers 4 and 3 likely dominate the ${ }^{10} \mathrm{~B}_{20}^{-}$population and the doublering 1 is likely absent from the PES measurement. The good match between the simulated and measured PESs based on the isomers 4 and 3 reinforces the prediction based on the energy calculations. The DFT calculations also show that the neutral double-ring $\mathrm{B}_{20}$ isomer has a large negative NICS value of -40 , indicating that 1 is strongly aromatic. Regarding to the accuracy of various functionals, we found that the PBE1PBE functional gives more consistent prediction on the relative stability than the B3LYP functional, in comparison with the high-level $a b$ initio calculations. Finally, the highlevel $a b$ initio calculations suggest that the planar-to-tubular structural transition starts at $\mathrm{B}_{20}$ for neutral clusters, but should occur beyond the size of $\mathrm{B}_{20}^{-}$for the anion clusters.

\section{ACKNOWLEDGMENTS}

The authors are grateful to Professor L. S. Wang for valuable discussions. One of the authors (X.C.Z.) acknowledges the support in part by grants from DOE (DE-FG0204ER46164), NSF (CHE, DMII, and MRSEC), John Simon Guggenheim Foundation, and the Nebraska Research Initiative, and by the Research Computing Facility and Bioinformatics Facility at University of Nebraska-Lincoln.

${ }^{1}$ I. Boustani, J. Solid State Chem. 133, 182 (1997).

${ }^{2}$ I. Boustani, Surf. Sci. 370, 355 (1997).

${ }^{3}$ I. Boustani and A. Quandt, Europhys. Lett. 39, 527 (1997).

${ }^{4}$ R. Saito, G. Dresselhaus, and M. S. Dresselhaus, Physical Properties of Carbon Nanotubes (Imperial College Press, London, 1998).

${ }^{5}$ J. Bai, X. C. Zeng, H. Tanaka, and J. Y. Zeng, Proc. Natl. Acad. Sci. U.S.A. 101, 2664 (2004).

${ }^{6}$ M. C. Daniel and D. Astruc, Chem. Rev. (Washington, D.C.) 104, 293 (2004).

${ }^{7}$ O. Mishima, J. Tanaka, S. Yamaoka, and O. Fukunaga, Science 238, 181 (1987).

${ }^{8}$ M. I. Eremets, V. V. Struzhkin, H. Mao, and R. J. Hemley, Science 293, 272 (2001).

${ }^{9}$ D. Meinkǒhn, Combust. Flame 59, 225 (1985).

${ }^{10}$ A. Demirbas, Energy Sources 27, 741 (2005).

${ }^{11}$ I. Boustani, Phys. Rev. B 55, 16426 (1997).

${ }^{12}$ Q. S. Li and H. W. Jin, J. Phys. Chem. A 106, 7042 (2002).

${ }^{13}$ A. K. Ray, I. A. Howard, and K. M. Kanal, Phys. Rev. B 45, 14247 (1992).

${ }^{14}$ L. Hanley, J. Witten, and S. L. Anderson, J. Phys. Chem. 92, 5803 (1988).

${ }^{15}$ H.-J. Zhai, A. N. Alexandrova, K. A. Birch, A. I. Boldyrev, and L.-S. Wang, Angew. Chem., Int. Ed. 42, 6004 (2003).

${ }^{16}$ H.-J. Zhai, B. Kiran, J. Li, and L.-S. Wang, Nat. Mater. 2, 827 (2003).

${ }^{17}$ B. Kiran, S. Bulusu, H.-J. Zhai, S. Yoo, X. C. Zeng, and L.-S. Wang, Proc. Natl. Acad. Sci. U.S.A. 102, 961 (2005).

${ }^{18}$ S. Chacko and D. G. Kanhere, Phys. Rev. B 68, 035414 (1997).

${ }^{19}$ I. Boustani, A. Rubio, and J. A. Alonso, Chem. Phys. Lett. 311, 21 (1999).

${ }^{20}$ M. A. L. Marques and S. Botti, J. Chem. Phys. 123, 014310 (2005).

${ }^{21}$ J.-I. Aihara, H. Kanno, and T. Ishida, J. Am. Chem. Soc. 127, 3698 (2005).

${ }^{22}$ C. L. Perkins, M. Trenary, and T. Tanaka, Phys. Rev. Lett. 77, 4772 (1996).

${ }^{23}$ H. Hubert, B. Devouard, L. A. J. Garvie, M. O’Keeffe, P. R. Buseck, W. T. Petuskey, and P. F. McMillan, Nature (London) 391, 376 (1998).

${ }^{24}$ M. J. Frisch, G. W. Trucks, H. B. Schlegel et al., GAUSSIAN 03, Revision C.02, Gaussian, Inc., Wallingford, CT, 2004.

${ }^{25}$ C. Møller and M. S. Plesset, Phys. Rev. 46, 618 (1934).

${ }^{26}$ M. Head-Gordon, J. A. Pople, and M. J. Frisch, Chem. Phys. Lett. 153, 503 (1988); M. J. Frisch, M. Head-Gordon, and J. A. Pople, ibid. 166, 
281 (1990); M. Head-Gordon and T. Head-Gordon, ibid. 220, 122 (1994); S. Saebo and J. Almlof, ibid. 154, 83 (1989).

${ }^{27}$ C. Lee, W. Yang, and R. G. Parr, Phys. Rev. B 37, 785 (1988); A. D. Becke, J. Chem. Phys. 98, 5648 (1993).

${ }^{28}$ J. P. Perdew, K. Burke, and M. Ernzerhof, Phys. Rev. Lett. 77, 3865 (1996); 78, 1396 (1997).

${ }^{29}$ Y. Zhao, B. J. Lynch, and D. G. Truhlar, Phys. Chem. Chem. Phys. 7, 43 (2005).

${ }^{30}$ T. H. Dunning, Jr., J. Chem. Phys. 90, 1007 (1989).

${ }^{31}$ K. Krishnan and J. A. Pople, Int. J. Quantum Chem. 14, 91 (1978); G. W. Trucks, E. A. Salter, C. Sosa, and R. J. Bartlett, Chem. Phys. Lett. 147, 359 (1988); G. W. Trucks, J. D. Watts, E. A. Salter, and J. Bartlett, ibid. 153, 490 (1988).

${ }^{32}$ J. A. Pople, R. Krishnan, H. B. Schlegel, and J. S. Binkley, Int. J. Quantum Chem. 14, 545 (1978); K. Raghavachari, G. W. Trucks, M. HeadGordon, and J. A. Pople, Chem. Phys. Lett. 157, 479 (1989).

${ }^{33}$ P. von R. Schleyer, C. Maerker, A. Dransfeld, H. Jiao, and N. J. R. van Eikema Hommes, J. Am. Chem. Soc. 118, 6317 (1996).

${ }^{34}$ J. A. Bohmann, F. Weinhold, and T. C. Tarrar, J. Chem. Phys. 107, 1173 (1997); E. D. Glendening, J. K. Badenhoop, A. E. Reed, J. E. Carpenter,
J. A. Bohmann, C. M. Morales, and F. Weinhold, NBO 5.0, Theoretical Chemistry Institute, University of Wisconsin, Madison, WI, 2001; http:// www.chem.wisc.edu/ nbo5

${ }^{35}$ W. An, Y. Gao, S. Bulusu, and X. C. Zeng, J. Chem. Phys. 122, 204109 (2005); S. Yoo and X. C. Zeng, ibid. 123, 164303 (2005).

${ }^{36}$ J. A. Pople, M. Head-Gordon, and K. Raghavachari, J. Chem. Phys. 87, 5968 (1987)

${ }^{37}$ M. Head-Gordon, R. J. Rico, M. Oumi, and T. J. Lee, Chem. Phys. Lett. 219, 21 (1994); M. Head-Gordon, D. Maurice, and M. Oumi, ibid. 246, 114 (1995).

${ }^{38}$ T. J. Lee and P. R. Taylor, Int. J. Quantum Chem., Symp. 23, 199 (1989).

${ }^{39}$ A. Hirsch, Z. Chen, and H. Jiao, Angew. Chem., Int. Ed. 39, 3915 (2000).

${ }^{40}$ C. S. Wannere, C. Corminboeuf, Z.-X. Wang, M. D. Wodrich, R. B. King, and P. Schleyer, J. Am. Chem. Soc. 127, 5701 (2005).

${ }^{41}$ R. B. King, T. Heine, C. Corminboeuf, and P. Schleyer, J. Am. Chem. Soc. 126, 430 (2004).

${ }^{42}$ J. Aihara and T. Tamaribuchi, Chem. Phys. Lett. 374, 104 (2003).

${ }^{43}$ T. Heine, P. Schleyer, C. Corminboeuf, G. Seifert, R. Reviakine, and J. Weber, J. Phys. Chem. A 107, 6470 (2003). 